\title{
An e-Delphi Study to Identify Priority Areas for Education on Advance Care Planning in COPD Management
}

\author{
Art Burgess Kelleher, Catherine Sweeney, Tony Foley, Ruth M Hally, Elaine Meehan, \\ Eileen Savage, Bettina Korn, and Nicola Cornally
}

\begin{abstract}
BACKGROUND: Individuals with COPD typically experience a gradual deterioration in health, with exacerbations and increased health-care utilization in the years before death. As such, adequate planning of future care is necessary. The objective of this research was to identify gaps in health-care professionals' knowledge related to advance care planning (ACP) for patients with COPD and to prioritize content of future educational interventions for health-care professionals accordingly. METHODS: A modified e-Delphi technique with 2 rounds of rating was used. After a literature review and expert consultation, a multidisciplinary panel developed 40 statements. Statements that did not achieve consensus in round 1 were re-presented in round 2, together with new statements developed after participant feedback. Health-care professionals with expertise in caring for people with COPD were asked to rate the importance of including each of the 40 topics in future educational interventions related to ACP and COPD by using a 5-point Likert scale. Average scores for each topic were calculated and expressed as percentages. Consensus on each topic was achieved if it obtained a score of $\geq \mathbf{8 0 \%}$. RESULTS: Thirty-eight health-care professionals were invited to participate, of whom, $21(55 \%)$ took part in both rounds. Consensus was achieved for 32 of 40 statements $(80 \%)$ in round 1 and for 5 of 10 statements $(50 \%)$ in round 2 . All statements relating to "COPD and palliative care" and "legislation for ACP" achieved consensus after one round. CONCLUSIONS: This study uncovered specific topics that related to ACP and COPD that healthcare professionals believe should be included in future educational interventions. In particular, there is a clear need for training that addresses the legal issues surrounding $\mathrm{ACP}$, and information on the best timing and content of ACP discussions with patients with COPD. Such training may enable health-care professionals to incorporate ACP into routine COPD management and, ultimately, improve patient care. Key words: advance care planning; chronic obstructive pulmonary disease; Delphi; educational needs assessment; training; education. [Respir Care 2020;65(3):347-354. (c) 2020 Daedalus Enterprises]
\end{abstract}

\section{Introduction}

COPD is a progressive lung disease that involves airway and/or alveolar abnormalities, and leads to a gradual

decline in lung function over time. Symptoms include chronic cough, sputum production, wheezing, dyspnea, and poor exercise tolerance. ${ }^{1}$ COPD is a leading cause of mortality worldwide, particularly among tobacco users and those exposed to significant levels of biomass fuels. ${ }^{2}$ As

\footnotetext{
Drs Burgess Kelleher, Sweeney, and Foley are affiliated with Faculty of Medicine and Health, School of Medicine, University College Cork, Cork, Ireland. Drs Hally, Meehan, Savage, and Cornally are affiliated with the Faculty of Medicine and Health, School of Nursing and Midwifery, University College Cork, Cork, Ireland. Ms Korn is affiliated with the Hospice Friendly Hospital Programme, St. James's Hospital, Dublin, Ireland.
}

The authors have disclosed no conflicts of interest.
Supplementary material related to this paper is available at http:// www.rcjournal.com.

Correspondence: Nicola Cornally PhD, School of Nursing and Midwifery, Brookfield Health Sciences Complex, University College Cork, College Road, T12 AK54, Cork, Ireland. E-mail: n.cornally@ucc.ie.

DOI: $10.4187 /$ respcare. 07072 
COPD progresses, many patients have a period of significant debility, with more frequent exacerbations and hospitalizations, increased symptom burden, and worsening quality of life. ${ }^{3}$ The natural history of COPD varies significantly among individuals, which makes it difficult to accurately predict the prognosis and likely disease trajectory. ${ }^{4}$ For this reason, health-care professionals who work with patients with COPD need to be familiar with, and proactive about, health-care planning as the disease progresses.

Advance care planning (ACP) refers to the process of discussing and reflecting with an individual patient about his or her goals, values, and preferences for future treatment in the event of deterioration that results in a loss of capacity to make and communicate decisions about his or her care preferences at the end of life. ${ }^{5}$ Results of studies show that ACP is associated with increased patient satisfaction with care and an increased probability that an individual will die in his or her preferred place. ${ }^{6-8}$ Due to the unpredictable nature of COPD and the associated difficulties in accurately predicting patients' disease trajectories, ACP may be particularly relevant for this patient group. There is evidence that the ACP needs of individuals with COPD are largely unmet, however, and patients and caregivers frequently put off having ACP discussions until such time that the patient deteriorates and treatment decisions are urgently required. ${ }^{4}$ To be most effective, ACP needs to be incorporated into routine management, and all health-care professionals involved in a patient's care need to have some level of familiarity with the ACP process. ${ }^{4}$ However, many health-care professionals find initiating ACP discussions difficult and believe that they have inadequate training in ACP and end-of-life discussions in general. ${ }^{9}$

An advance care plan is a written record of the outcome of an ACP discussion. Various types of ACP forms exist, and they address issues such as treatment decisions, place of care, finances, and after-death preferences. Examples include Think Ahead, an initiative of the Irish Hospice Foundation (https://hospicefoundation.ie/programs/publicawareness/think-ahead/. Accessed March 23, 2019); Five Wishes, a program of aging with dignity (https://five wishes.org/. Accessed March 23, 2019); and Respecting Choices (https://respectingchoices.org. Accessed March 23, 2019). Although an advance care plan is a way to formalize an ACP conversation into a record that can be used to inform decision making at end of life, it is not legally binding. An advance care plan must be converted into an advance directive or similar, depending on the legislation of the governing state and/or country, for it to be legally binding. ${ }^{10}$ For example, in Ireland, an advance care plan is not legally binding unless it is in the form of an advance health-care directive, also known as a living will.

\section{QUICK LOOK}

\section{Current knowledge}

As COPD progresses, many patients experience more frequent exacerbations and hospitalizations, increased symptom burden, and worsening quality of life. Advance care planning (ACP) may increase patients' levels of satisfaction with care, but the ACP needs of individuals with COPD are largely unmet. All healthcare professionals involved in a patient's care need to have some level of familiarity with the ACP process; however, many believe that they have inadequate training in ACP and end-of-life discussions in general.

\section{What this paper contributes to our knowledge}

Health-care professionals from a range of backgrounds were largely in agreement about the topics that should be included in future educational interventions on the topic of ACP for individuals with COPD. In addition to covering the legal issues that surround ACP, future educational interventions should provide health-care professionals with additional knowledge and strategies to assist with identifying patients with COPD who are suitable for palliative care and ACP discussions, as well as appropriate timing and content of such conversations.

With an aging population and the signing into law of the Assisted Decision Making (Capacity) Act 2015, ${ }^{11}$ there is a growing recognition in Ireland that health-care professionals will need to be familiar with the legislation concerning ACP as well as its implications. At the time of this writing, the aforementioned Act ${ }^{11}$ has yet to be fully enacted, and there is a lack of clarity around the meaning of the term, "capacity." In addition to concerns about capacity and the legal implications of ACP, other barriers to initiating ACP discussions include a lack of time, a fear of patient reaction, and unwillingness on the part of patients or families to accept the diagnosis and/or prognosis. ${ }^{12}$ Of particular relevance to patients with COPD is the difficulty that health-care professionals face in identifying the right time to initiate a conversation about $\mathrm{ACP}$ with a patient and his or her family. ${ }^{13}$

To date, ACP research has focused primarily on other groups, particularly patients with cancer, ${ }^{14,15}$ and there is a paucity of research with regard to ACP for patients with COPD. A lack of training for health-care professionals has been identified as a barrier to implementing ACP into routine management. ${ }^{16,17}$ To ensure that the topics of future educational interventions are relevant and meaningful to the target audience, the objective of this study was to identify gaps in health-care professionals' knowledge re- 
lated to ACP for patients with COPD and to prioritize content of future educational interventions for health-care professionals accordingly.

\section{Methods}

\section{Funding}

This study was supported by a GlaxoSmithKline Independent Medical Education grant. GlaxoSmithKline had no input in the design of the study, data collection, data analysis or interpretation, or in the preparation, review, or approval of this manuscript.

\section{Study Design}

This study was based on the Delphi process, which is a method of achieving a consensus of opinion associated with a specific topic through a series of structured questionnaires (known as rounds). ${ }^{18}$ The Delphi process generally includes the following stages: (1) recruit relevant experts, (2) compile a questionnaire with a list of statements related to the topic that the experts are asked to rate for agreement, (3) calculate the level of agreement with each statement, and (4) give anonymous feedback to the participants about how their responses compared with others' responses and provide them with the opportunity to revise their responses in light of this feedback. ${ }^{18}$ This process continues until consensus is reached.

The Delphi method uses many factors to assess the extent of agreement on a given topic or issue. These factors include independence of decisions (through responses to anonymous questionnaires), decentralization (the members of the group operate autonomously but share decisions through the facilitator), and aggregation (through the facilitator's organization of the group and statistical summarization of results). ${ }^{18,19}$ Diversity of expertise is not a requirement of the Delphi method but diversity of panel members does promote better decision making. ${ }^{20}$ For this study, an e-Delphi method was selected in an effort to capture a number of experts across a range of disciplines in diverse geographic locations.

\section{Preparation for the e-Delphi Process}

For this study, a modified e-Delphi method with 2 rounds of rating was used. First, to summarize the available literature on current practices about ACP for individuals with COPD, a scoping review of the literature that reported on original research in the past 10 years was conducted by following the framework of Arksey and O'Malley. ${ }^{21}$ The research team, including 4 experienced health-care professionals from medical $(n=2)$ [TF, CS], nursing $(n=1)[\mathrm{NC}]$, and physiotherapy $(n=1)$ back- grounds, prepared 40 statements that aligned to 3 separate subject areas related to ACP and COPD: (1) ACP discussions and documentation in COPD care, (2) COPD and palliative care, and (3) legislation for ACP. These statements were informed by the clinical experience of the research team and the findings of the scoping literature review. Opinion was also sought from an international expert on the topic.

An online questionnaire, by using the LimeSurvey platform (LimeSurvey, Hamburg, Germany), was developed (https://www.limesurvey.org/. Accessed March 23, 2019). LimeSurvey is an interactive web application for creating, managing, and monitoring online surveys. It is secure, and survey data are stored within the host university's information technology infrastructure, which ensures that privacy of data is maintained. The questionnaire included demographic questions and 40 statements under thematically aligned subject headings. The participants were asked to rate the importance of each topic for inclusion in future educational interventions for health-care professionals with regard to ACP for patients with COPD.

This rating was based on a 5-point Likert scale (1 [very limited importance], 2 [limited importance], 3 [important but not critical], 4 [critical], and 5 [very critical]). An average score for each statement was calculated and is expressed as a percentage. For this study, consensus was considered to have been reached when a statement achieved a score of $\geq 80 \%$ (this was equivalent to an average score of 4). There is no universally accepted threshold for defining consensus as part of the Delphi process. The level of percentage agreement necessary to reach consensus for this study was informed by the literature on consensus criterion in Delphi studies, ${ }^{22}$ the previous experience of the research team, ${ }^{23}$ and by Delphi studies in similar research areas. ${ }^{24,25}$ The option to provide free-text comments was provided throughout the questionnaire to give the participants the opportunity to indicate areas not covered in the statements provided or to elaborate on particular topic areas.

\section{Recruitment and Participation}

Members of the research team each identified up to 10 health-care professionals from their respective fields to participate in the e-Delphi process. The participants were purposively selected based on their known expertise in the area, and they needed to be experienced health-care professionals with medical, nursing, or allied health backgrounds. The eligible participants $(N=37)$ were invited by using e-mail and were provided with an information leaflet with regard to participation before beginning the online survey.

Round 1 was open for 2 weeks, after which the responses were analyzed to determine if consensus had been achieved. For the open-ended responses, the facilitator un- 


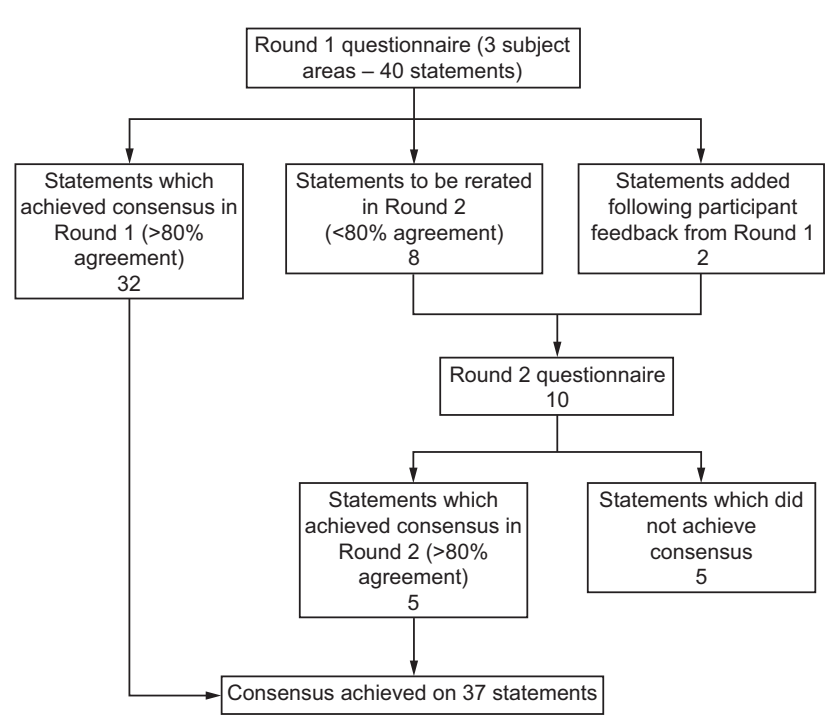

Fig. 1. Flow chart, showing the number of statements and outcomes of each e-Delphi round of rating.

dertook thematic analysis of the participants' comments and compared them with existing statements. This step of the process gave rise to new statements, which were presented to the research team, who supported or rejected their inclusion in round 2 based on relevance and alignment to similar statements.

For round 2, the participants were re-presented with the statements that did not achieve consensus in round 1 , together with the new statements that were generated from participants' comments in round 1 . When re-rating statements in round 2, the participants were also provided with their original score for that statement in round 1 as well as the average group score from all the participants for that statement. Round 2 statements were scored on the same 5-point Likert scale as in round 1. Consensus for round 2 was also set at $80 \%$ participant agreement. All statements that scored $\geq 80 \%$ were included, whereas any statements that failed to achieve this level of agreement were excluded. Round 2 was also open for 2 weeks. The modified Delphi method used in this study is outlined in Figure 1.

\section{Data Analysis}

The participants' demographic data were summarized by using descriptive statistics. After each round of the Delphi process, descriptive statistics were generated to include mean scores and percentage consensus rates for each statement. Qualitative data in the form of participants' comments from round 1 were analyzed thematically and compared with existing statements. New statements were written based on these comments. The study was conducted at Brookfield Health Sciences Complex, Uni-
Table 1. Participant Characteristics

\begin{tabular}{lc}
\hline \hline \multicolumn{1}{c}{ Characteristic } & Results, $n(\%)$ \\
\hline Sex & \\
Male & $6(28.6)$ \\
Female & $14(66.7)$ \\
Other/unknown & $1(4.7)$ \\
Age & \\
$21-30$ y & $3(14.3)$ \\
$31-40$ y & $5(23.8)$ \\
$41-50$ y & $5(23.8)$ \\
$51-60$ y & $5(23.8)$ \\
$>60$ y & $3(14.3)$ \\
Years of experience & \\
$0-10$ y & $4(19.0)$ \\
$11-20$ y & $5(23.8)$ \\
$21-30$ y & $6(28.6)$ \\
Current yorkplace & $6(28.6)$ \\
Hospital & \\
Hospice & $12(57.1)$ \\
General practice & $4(19.0)$ \\
Other community setting & $4(19.0)$ \\
\hline$N=21$. & $1(4.9)$ \\
\hline
\end{tabular}

versity College Cork, College Road, Cork, Ireland. Ethics approval for this study was granted by University College Cork's social research ethics committee.

\section{Results}

\section{Overview of the Participants}

Thirty-eight health-care professionals were invited to participate in this study, of whom, $21(55 \%)$ participated in round 1 and round 2. Of the 21 participants, $14(67 \%)$ were female. Twelve participants $(57 \%)$ had $>20$ years of clinical experience. Most of the participants worked in a hospital setting $(n=12)$, with the remainder distributed across general practice $(n=4)$, hospice $(n=4)$, and the community $(n=1)$ (Table 1$)$.

\section{Summary of the Rating Process}

The number of statements in each round of the process, including a breakdown of how many statements were accepted, excluded, and re-rated, are outlined in Table 2.

\section{Round 1}

Forty statements were included for rating in round 1, from which 32 (80\%) achieved consensus (Table 2). This included all 12 statements that were related to "COPD and 
Table 2. Number of Statements Accepted, Rejected, and Re-Rated at Each Stage

\begin{tabular}{lccccc}
\hline \hline Round & $\begin{array}{c}\text { Total } \\
\text { Statements }\end{array}$ & $\begin{array}{c}\text { Statements } \\
\text { Accepted }\end{array}$ & $\begin{array}{c}\text { Statements } \\
\text { Excluded }\end{array}$ & $\begin{array}{c}\text { Statements } \\
\text { to Be } \\
\text { Re-Rated }\end{array}$ & $\begin{array}{c}\text { New } \\
\text { Statements } \\
\text { Added }\end{array}$ \\
\hline 1 & 40 & 32 & 0 & 8 & 2 \\
2 & 10 & 5 & 5 & 0 & N/A \\
\multicolumn{2}{ll}{ N/A = not applicable } & & & & \\
\hline
\end{tabular}

palliative care" and all 8 statements that were related to "legislation for ACP." With regard to the statements related to "ACP discussions and documentation in COPD care," consensus was achieved for 12 of 20 statements (60\%) in round 1 (see the supplementary materials at http:// www.rcjournal.com for detailed participant responses to the statements included in round 1).

\section{Round 2}

The 8 statements that did not achieve consensus in round 1 were included in round 2 . In addition, the initial expert panel agreed on the inclusion of 2 new statements that were formulated based on the participants' comments in round 1 . The first of these related to comorbidities likely to be experienced by patients with COPD, and the second was based on the need for a person-centered approach to care. Of the 10 statements included in round 2 , consensus was achieved for $5(50 \%)$. This included the 2 statements that were formulated from the feedback obtained in round 1. The participants' scores for 3 of the statements that did not achieve consensus in round 1 changed sufficiently, such that these statements achieved consensus in round 2 . (Table 3.)

\section{Priority Topics}

Among the topics identified as priorities by the participants were identifying patients with COPD who could benefit from palliative care interventions, recognizing the appropriate time to begin palliative care, identification of changes in care needs for patients with COPD, addressing mismatched patient or family expectations, strategies for conversations with patients with poor prognosis, and strategies for communicating outcomes of ACP discussions to the wider health-care team, all of which reached $85 \%$ agreement in round 1. In addition, the statements, "professional responsibilities arising from the Assisted Decision-Making (Capacity) Act 2015" and "Code of Practice for $A C P$ " both reached $90 \%$ agreement in round 1.

\section{Discussion}

The 37 statements that achieved consensus provided an overview of the core areas of content related to ACP that need to be included in future training initiatives for healthcare professionals who care for people with COPD. The high rate of acceptance of most of the statements included in this e-Delphi study highlighted the range of areas in which there is a perceived need for further education.

All 12 statements related to COPD and palliative care achieved consensus after one round of rating, which highlights the widespread support for specific emphasis to be placed on COPD in future ACP research. The statement with the highest level of agreement (91\%) called for emphasis to be placed on identifying patients with COPD who could benefit from palliative care interventions. This was followed closely by recognizing the appropriate time to begin palliative care and identification of changes in care needs for patients with COPD, each with $90 \%$ agreement. This feedback echoes the findings of Spence et al, ${ }^{26}$ which noted that health-care professionals are often unsure of the best time to introduce palliative care into the care pathway for patients with COPD.

In addition, guidance about who is responsible for initiating ACP is often lacking, and a lack of established protocols for who is responsible for initiating ACP has been identified as another barrier to implementing COPD into routine COPD management. ${ }^{16,17,26}$ For patients, the key is having a well-informed health-care professional who is familiar with them and their history to carry out ACP discussions at an appropriate time. ${ }^{3,27}$ For some, this may be a general practitioner, but, for others, it may be a hospital-based treating clinician. This highlights the fact that all health-care professionals involved in the care of patients with COPD need to have some level of familiarity with the process of $\mathrm{ACP}$, and the resounding agreement with statements in this section confirmed the need for guidance on both the initiation and continuation of ACP throughout the COPD journey.

All statements related to legislation for ACP were accepted after one round, which highlighted another priority area for those working in this field. The focus on legislation for ACP likely reflects a growing awareness of the forthcoming enactment of legislation on the topic in Ireland in the form of the Assisted Decision-Making (Capacity) Act 2015. ${ }^{11}$ This may not be a priority education need in other countries where legislation is clearer and well established.

It is also important to consider the statements that did not achieve consensus. These statements focused primarily on the theory of ACP, the difference between advance care plans and advance health-care directives, types of ACP, and current ACP research in COPD. One possible explanation for this is that these topics are not as relevant to the 


\section{Advance Care Planning for COPD}

Table 3. Rounds 1 and 2, Percentage Agreement to Statements, Presented According to Subject Area

\begin{tabular}{|c|c|c|}
\hline \multirow{2}{*}{ Statement } & \multicolumn{2}{|c|}{ Agreement, \% } \\
\hline & Round 1 & Round 2 \\
\hline \multicolumn{3}{|l|}{ ACP discussions and documentation in COPD care } \\
\hline Discussing ACP with family members of patients with COPD & 82 & NA \\
\hline Family and caregivers needs & 82 & NA \\
\hline Addressing mismatched patient or family expectations & 90 & NA \\
\hline Incorporating $\mathrm{ACP}$ into routine management of $\mathrm{COPD}$ & 81 & NA \\
\hline Do-not-resuscitate orders as part of ACP in patients with COPD & 82 & NA \\
\hline Clarifying goals of treatment, eg, palliative vs curative & 80 & NA \\
\hline Strategies for conversations with patients with poor prognosis & 89 & NA \\
\hline Timing and frequency of ACP discussions & 81 & NA \\
\hline General principles of palliative care & 86 & NA \\
\hline Palliative care competencies (profession specific) & 85 & NA \\
\hline Strategies for communicating outcomes of ACP discussions to the wider health-care team & 89 & NA \\
\hline The benefits of ACP in COPD care & 82 & NA \\
\hline The key difference between ACP and advance directives & 74 & $78^{*}$ \\
\hline Discussing life expectancy & 79 & 80 \\
\hline General principles regarding discussions about prognosis & 78 & 86 \\
\hline Revision of ACP decisions and documentation & 79 & 81 \\
\hline $\begin{array}{l}\text { Physician-patient scenario and role play simulations about ACP discussions and communication } \\
\text { techniques for initiating conversations around ACPs }\end{array}$ & 68 & $69^{*}$ \\
\hline Theoretical perspectives on ACP & 69 & $61^{*}$ \\
\hline Types of advance care directives & 79 & $74 *$ \\
\hline Current research on $\mathrm{ACP}$ in $\mathrm{COPD}$ & 77 & $74 *$ \\
\hline \multicolumn{3}{|l|}{ COPD and palliative care } \\
\hline Identifying patients with COPD who could benefit from palliative care interventions & 91 & NA \\
\hline Recognizing the appropriate time to begin palliative care & 90 & NA \\
\hline Identification of changes in care needs for patients with COPD & 90 & NA \\
\hline Advantages of ACP for patients with COPD & 88 & NA \\
\hline Patients with COPD preference of ACP discussions & 83 & NA \\
\hline Barriers to initiation of ACP for patients with COPD & 83 & NA \\
\hline Identification of COPD transition points for ACP discussions & 84 & NA \\
\hline Typical disease trajectories for people with COPD & 82 & NA \\
\hline Factors assisting prognostication in the patient with COPD & 81 & NA \\
\hline Predictors of mortality during and after exacerbations of COPD & 81 & NA \\
\hline Learning opportunities for a team from the death of a patient with COPD & 83 & NA \\
\hline Self-care strategies for HCPs engaged in ACP with patients and families & 82 & NA \\
\hline Legislation for ACP & & NA \\
\hline Professional responsibilities arising from the Assisted Decision-Making (Capacity) Act 2015 & 90 & NA \\
\hline Code of Practice for HCPs for ACP & 90 & NA \\
\hline Guiding principles of the Assisted Decision-Making (Capacity) Act 2015 & 87 & NA \\
\hline Exploration of the key themes in the Assisted Decision-Making (Capacity) Act, 2015 & 87 & NA \\
\hline $\begin{array}{l}\text { Review of Ireland's Health Service Executive recommendations in relation to the interpretation and } \\
\text { operation of the advance health-care directives provisions contained in the Act }\end{array}$ & 85 & NA \\
\hline Understanding what makes ACP a legal document & 84 & NA \\
\hline Do-not-resuscitate orders as part of ACP in patients with COPD & 89 & NA \\
\hline Ethics issues involved in ACP & 92 & \\
\hline \multicolumn{3}{|l|}{ Statements formulated after round 1 feedback } \\
\hline $\begin{array}{l}\text { Exploration of comorbidities (eg, ischemic heart disease and osteoporosis), which may impact on } \\
\text { the patient with advanced COPD }\end{array}$ & NA & 84 \\
\hline $\begin{array}{l}\text { A person-centered approach to advance care planning for persons with COPD (eg, values } \\
\text { discussion, person-focused outcomes) }\end{array}$ & NA & 86 \\
\hline \multicolumn{3}{|l|}{$\begin{array}{l}N=21 . \\
* \text { Did not achieve consensus after } 2 \text { rounds. } \\
\text { ACP = advance care planning } \\
\mathrm{NA}=\text { not applicable } \\
\mathrm{HCP}=\text { health-care professional }\end{array}$} \\
\hline
\end{tabular}


everyday practices of health-care professionals. There may also be a perception that they increase the complexity in an area that is already perceived to be complex. Of note, most participants in this e-Delphi study $(65 \%)$ had $>20$ years of experience in their field, so it is possible that these topics are no longer a top priority for these experienced individuals but may be of interest to less-experienced health-care professionals working in this area.

One of the main reasons for using the Delphi method in this study was to provide the participants with the opportunity to provide feedback. The research team believed that there might be other topics that health-care professionals would deem important to be included in future educational interventions but which were not readily identifiable from the available literature. Two new statements were developed as part of the process. The first area identified by the participants was the impact that COPD comorbidities may have on future care. It is accepted that comorbidities contribute to the severity of COPD and need to be taken into account when planning a patient's management approach. ${ }^{2}$

The most common COPD comorbidities include other respiratory conditions (eg, asthma, lung cancer), cardiovascular conditions (eg, hypertension, coronary heart disease), metabolic comorbidities (eg, osteoporosis), and mental comorbidities (eg, anxiety, depression). ${ }^{28}$ When comorbidities are present, a holistic approach to care is required, with health-care professionals involved in COPD management working together with professionals specialized in the management of other diseases to provide multidisciplinary care for patients. ${ }^{28}$ The participants also identified the importance of keeping the patient at the center of the ACP approach. This, along with the strong support for a focus on ethics issues, reflects an interest in identifying and supporting the needs and wants of the patient.

The use of the modified e-Delphi method is one of the main strengths of this study because it is particularly appropriate when developing consensus when existing evidence is insufficient. The e-Delphi method provides the participants with anonymity and avoids issues of group conformity, influences of dominant personalities, prestige, and politics. ${ }^{29}$ Diversity needs to be taken into consideration when carrying out a Delphi process, ${ }^{18,19}$ and this study was strengthened by including participants from a range of professional backgrounds and who work across various settings. There has been some debate with regard to the validity of the Delphi method. ${ }^{30}$ However, these critiques are mainly focused on poor quality studies rather than the Delphi method itself. It has been recommended that results be matched to observable events, but there is minimal literature with which to compare the results of this study. Researcher influence when deciding on the initial statements to include is a potential limitation to the Delphi method. Using the findings of a review of the literature as well as an expert panel to decide on statements to include helped to minimize these risks.

Internet-based research methods, for example, the e-Delphi method, are increasing in popularity and help to reach participants from diverse locations. They are associated with convenience for participants, time and cost savings, and multiple data management options. They have a number of limitations, however, including internet accessibility challenges and technological difficulties. ${ }^{31} \mathrm{We}$ did not experience these challenges in the current study. A limitation of this study was the small number of participants. However, as few as 10 members have been reported to yield strong evidence in previous Delphi studies. ${ }^{32}$

This study was carried out over the academic summer break, which may have affected response rates. Each round was kept open for 2 weeks. Given the time of year, it may have been beneficial to leave each round open for longer. This was not feasible due to time constraints. The choice of methodology prevented an in-depth discussion among the expert participants; however, the ability to provide responses to the open-ended questions allowed the participants to share their ideas and knowledge with the research team. In addition, regular meetings of the research team facilitated in-depth discussion on selected topics as required.

\section{Conclusions}

This study included participants from a range of healthcare disciplines, but consensus was achieved for most statements after one round of rating, which demonstrated widespread agreement on the topics that should be included in future educational interventions on the topic of ACP for individuals with COPD. In addition to covering the legal issues surrounding ACP, future educational interventions should provide health-care professionals with additional knowledge and strategies to assist with identifying patients with COPD who are suitable for palliative care as well as the most appropriate timing and content of such discussions. Information from this study should be used to inform the content of future education and training initiatives, and, in time, this content should be evaluated to ensure that it remains relevant going forward. Another important step is to consider input from people living with COPD and their carers before the rollout of any educational interventions.

\section{ACKNOWLEDGMENTS}

The authors thank all members of the CONCORDAT (Incorporating ACP into Routine COPD Management) research team based at University College Cork, Marymount University Hospital and Hospice, The All Ireland Institute of Hospice and Palliative Care, The Irish Hospice Foundation and St. James's Hospital, Dublin, from which this research is part of a larger program of research on ACP in COPD. The authors thank the 


\section{Advance Care Planning for COPD}

health-care professionals who gave their time to participate in the eDelphi rating process. They also thank Karen Detering from Advance Care Planning Australia for her expert advice.

\section{REFERENCES}

1. Qaseem A, Wilt TJ, Weinberger SE, Hanania NA, Criner G, van der Molen T, et al.; American College of Physicians; American College of Chest Physicians, American Thoracic Society; European Respiratory Society. Diagnosis and management of stable chronic obstructive pulmonary disease: a clinical practice guideline update from the American College of Physicians, American College of Chest Physicians, American Thoracic Society, and European Respiratory Society. Ann Intern Med 2011;155(3):179-191.

2. Global Strategy for the Diagnosis, Management and Prevention of COPD, Global Initiative for Chronic Obstructive Lung Disease (GOLD) 2017. Available from: https://goldcopd.org. Accessed November 11, 2019.

3. Seamark DA, Seamark CJ, Halpin DM. Palliative care in chronic obstructive pulmonary disease: a review for clinicians. J R Soc Med 2007;100(5):225-233.

4. Detering KM, Sutton EA, McDonald CF. Recognising advanced disease, advance care planning and recognition of dying for people with COPD. In: Bausewein C, Currow DC, Johnson MJ, editors. Palliative care in respiratory disease (ERS Monograph). Switzerland, Lausanne: European Respiratory Society; 2016:204-220.

5. Janssen DJ, Engelberg RA, Wouters EF, Curtis JR. Advance care planning for patients with COPD: past, present and future. Patient Educ Couns 2012;86(1):19-24.

6. Abel J, Pring A, Rich A, Malik T, Verne J. The impact of advance care planning of place of death, a hospice retrospective cohort study. BMJ Support Palliat Care 2013;3(2):168-173.

7. Detering KM, Hancock D, Reade MC, Silvester W. The impact of advance care planning on end of life care in elderly patients: randomised controlled trial. BMJ 2010;340:c1345.

8. Curtis JR, Wenrich MD, Carline JD, Shannon SE, Ambrozy DM, Ramsey PG. Patients' perspectives on physician skill in end-of-life care: differences between patients with COPD, cancer, and AIDS. Chest 2002;121(1):356-362.

9. Knauft E, Nielsen EL, Engelberg RA, Patrick DL, Curtis JR. Barriers and facilitators to end-of-life care communication for patients with COPD. Chest 2005;127(6):2188-2196.

10. Castillo LS, Williams BA, Hooper SM, Sabatino CP, Weithorn LA, Sudore RL. Lost in translation: the unintended consequences of advance directive law on clinical care. Ann Intern Med 2011;154(2): 121-128.

11. The Assisted Decision-Making (Capacity) Act 2015 (Commencement of Certain Provisions) (No. 2) Order 2016. (Ireland)

12. Howard M, Bernard C, Klein D, Elston D, Tan A, Slaven M, et al. Barriers to and enablers of advance care planning with patients in primary care: survey of health care providers. Can Fam Physician 2018;64(4):e190-e198.

13. Aslakson RA, Reinke LF, Cox C, Kross EK, Benzo RP, Curtis JR. Developing a research agenda for integrating palliative care into critical care and pulmonary practice to improve patient and family outcomes. J Palliat Med 2017;20(4):329-343.

14. O'Sullivan EM, Higginson IJ. 'I'll continue as long as I can, and die when I can't help it': a qualitative exploration of the views of end- of-life care by those affected by head and neck cancer (HNC). BMJ Support Palliat Care 2016;6(1):43-51.

15. De Vleminck A, Pardon K, Beernaert K, Deschepper R, Houttekier $\mathrm{D}$, Van Audenhove C, et al. Barriers to advance care planning in cancer, heart failure and dementia patients: a focus group study on general practitioners' views and experiences. PLoS One 2014;9(1): e84905.

16. Gaspar C, Alfarroba S, Telo L, Gomes C, Bárbara C. End-of-life care in COPD: a survey carried out with Portuguese pulmonologists. Rev Port Pneumol 2014;20(3):123-130.

17. Ecenarro PS, Iguiñiz MI, Tejada SP, Malanda NM, Imizcoz MA, Marlasca LA, Navarrete BA. Management of COPD in end-of-life care by Spanish pulmonologists. COPD 2018;15(2):171-176.

18. Jorm AF. Using the Delphi expert consensus method in mental health research. Aust N Z J Psychiatry 2015;49(10):887-897.

19. Jones J, Hunter D. Consensus methods for medical and health services research. BMJ 1995;311(7001):376-380.

20. Page SE. The difference: how the power of diversity creates better groups, firms, schools, and societies. Princeton: Princeton University Press; 2008.

21. Arksey H, O’Malley L. Scoping studies: towards a methodological framework. Int J Soc Res Methodol 2005;8(1):19-32.

22. Keeney S, Hasson F, McKenna H. Consulting the oracle: ten lessons from using the Delphi technique in nursing research. J Adv Nurs 2006;53(2):205-212

23. Jennings AA, Guerin N, Foley T. Development of a tool for monitoring the prescribing of antipsychotic medications to people with dementia in general practice: a modified eDelphi consensus study. Clin Interv Aging 2018;13:2107-2117.

24. van der Steen JT, Radbruch L, Hertogh CM, de Boer ME, Hughes JC, Larkin P, et al.; European Association for Palliative Care (EAPC). White paper defining optimal palliative care in older people with dementia: a Delphi study and recommendations from the European Association for Palliative Care. Palliat Med 2014;28(3):197-209.

25. Downing J, Knapp C, Muckaden MA, Fowler-Kerry S, Marston J, ICPCN Scientific Committee. Priorities for global research into children's palliative care: results of an International Delphi Study. BMC Palliat Care 2015;14(1):36.

26. Spence A, Hasson F, Waldron M, Kernohan WG, McLaughlin D, Watson B, et al. Professionals delivering palliative care to people with COPD: qualitative study. Palliat Med 2009;23(2):126-131.

27. Brown M, Brooksbank MA, Burgess TA, Young M, Crawford GB. The experience of patients with advanced chronic obstructive pulmonary disease and advance care-planning: a South Australian perspective. J Law Med 2012;20(2):400-409.

28. Hillas G, Perlikos F, Tsiligianni I, Tzanakis N. Managing comorbidities in COPD. Int J Chron Obstruct Pulmon Dis 2015;10:95-109.

29. Dalkey NC. (1969) The Delphi method: an experimental study of group opinion. The RAND corporation: Santa Monica, USA.

30. Sackman H. Delphi Critique: Expert Opinion, Forecasting and Group Process. Lexington, Massachusetts: Lexington Books; 1975.

31. Donohoe H, Stellefson M, Tennant B. Advantages and limitations of the e-Delphi technique: Implications for health education researchers. Am J Health Educ 2012;43(1):38-46.

32. Skulmoski GJ, Hartman F, Krahn J. The Delphi method for graduate research. J Inf Technol Educ 2007;6. 